\title{
Optical Coherence Tomography Angiography Compared to Fluorescein Angiography in Central Retinal Vein Occlusion
}

\author{
Khaled Selim*, Mostafa Mahmoud* and Ali Maher Ibrahim El-Shaarawy** \\ * Department of Ophthalmology, Faculty of Medicine, Al-Azhar University \\ **Memorial Institute of Ophthalmic Research \\ Corresponding Author: drali_sha3rawy@yahoo.com
}

\begin{abstract}
Background: Retinal vein occlusion is the second most common cause of retinal vascular disease worldwide after diabetic retinopathy.

Objective: It is a prospective comparative study to evaluate the optical coherence tomography angiography appearance of superficial and deep capillary plexa in eyes with central retinal vein occlusion in comparison to conventional finding in fluorescein angiography. The study was approved by the medical ethics committee of Al-Azhar University Hospitals and a written informed consent is obtained from all patients.

Patients and Methods: A total of 19 eyes of 17 patients (with a known clinical diagnosis of CRVO; any stage, any type), were recruited. All patients underwent standard ophthalmological examination together with multimodal imaging procedures including; color fundus photography, fluorescein angiography and optical coherence tomography angiography. Quantitative flow analysis of the SCP has been performed for selected 15 images of 15 eyes with CRVO versus a single eye of a normal control.

Results: Optical coherence tomography angiography can qualitatively demonstrate all the macular complications of CRVO including; ischemia, capillary dilation, microaneurysms, collateral vessels, edema and intraretinal hemorrhages, with superiority to fluorescein angiography especially in the presence of retinal hemorrhages and retinal edema. OCTA with the help of the co-registered en face OCT images, can demonstrate laser scars, at the level of the choriocapillaris and can help in detecting disc edema. Statistical analysis of the incidence of the different pathological findings in CRVO, has revealed more severe affection of the deep capillary plexus than the superficial one.

Conclusion: OCTA allows stratigraphic analysis of the retinal and choroidal vasculatures. It also allows performing quantitative flow analysis of the retinal vasculature providing an easily interpretable noninvasive objective tool for the management and monitoring the progression of CRVO.
\end{abstract}

Keywords: Central Retinal vein occlusion, Optical coherence tomography angiography, Fluorescein angiography.

\section{INTRODUCTION}

Retinal vein occlusion is the second most common cause of retinal vascular disease worldwide after diabetic retinopathy (1) and around 6 million of people in the world have retinal vein occlusion (RVO) ${ }^{(2)}$.

The commonly accepted pathophysiology of central and branch retinal venous occlusion is thrombosis ${ }^{(3)}$ resulting in elevated venous pressure and consequent macular edema, retinal ischemia, and rupture of the retinal vessel wall with intraretinal hemorrhage. In longstanding cases, after absorption of the intraretinal hemorrhage, there are several retinal vascular abnormalities, such as retinal ischemic changes in the form of capillary nonperfusion, capillary dilation, microaneurysms, telangiectatic vessels, and collateral circulation ${ }^{(4)}$. Diagnosis of retinal vein occlusion can be achieved clinically or by investigations including fundus fluorescein angiography (FFA), optical coherence tomography (OCT) and the recent optical coherence tomography angiography (OCTA).

Fluorescein Angiography has been the gold standard for the diagnosis of various vascular diseases. It is particularly useful in highlighting the retinal perfusion especially that of the macular area including visualizing vascular filling defects, areas of capillary nonperfusion, and retinal vessel tortuosity, dilation, aneurysms, and leakage ${ }^{(5)}$.

However, fluorescein angiography does not image the radial peripapillary or the 
deep capillary networks well (6). Optical coherence tomography angiography is a novel method that measures depth resolved blood flow in the retina using the variance in the intensity and phase of the reflected OCT beam. The angiograms are acquired using a spectral domain-optical coherence tomography (SDOCT) hardware configuration and modified acquisition with motion correction technology (3). The newly developed amplitude decorrelation algorithm which is called the split-spectrum amplitude decorrelation algorithm (SSADA) provides information about blood flow by comparing two consecutive B Scans ${ }^{(7)}$.

Simultaneously evaluate both macular perfusion and edema. For the first time an imaging technique enable the evaluation of deep capillary plexus, which appears to be affected than the superficial capillary plexus in $\mathrm{CRVO}^{(7)}$.

\section{PATIENTS AND METHODS}

This prospective comparative study was carried out on patients attending the Ophthalmology Department of Al-Azhar University Hospitals and Memorial Institute of Ophthalmology at Giza. All participant names were hidden and replaced by code numbers to maintain privacy of them.

A total of 20 eyes (with a known clinical diagnosis of CRVO; any stage, any type), were subjected to standard ophthalmological examination together with multimodal imaging procedures including; color fundus photography, fluorescein angiography and optical coherence tomography angiography. All imaging procedures were performed on the same day or during the same week without in-between intraocular injections or laser treatment. Quantitative flow analysis of the superficial capillary plexuses (SCP) was performed for selected 15 images of 15 eyes with CRVO versus a single eye of a normal control.

\section{(1) Inclusion criteria:}

(1) Patients with a known clinical diagnosis of CRVO; any stage, any type. (2) Patients with CRVO; whether, it is associated or not with systemic hypertension, and /or glaucoma and whether, it is previously treated or not.

\section{(2) Exclusion criteria:}

1) Patients with advanced macular pathologies. (2) Diabetic patients (3) Patients with poor quality images, either on OCT angiography (Mainly due to eye movements) or on FA (Mainly due to media opacities).

\section{MATERIALS AND METHODS:}

For all patients, a standard clinical examination and testing as appropriate for their clinical disease was done including:

- History taking including; personal history, history of systemic diseases particularly; diabetes mellitus and hypertension, history of ocular diseases; mainly glaucoma, history of previous treatments; mainly retinal laser photocoagulation and/or intraocular injections).

- BCVA (using Snellen's visual acuity chart).

- Pupillary light reflex.

- IOP measurement (using Goldmann applanation tonometer).

- Slit lamp examination of the anterior segment.

- Gonioscopy (Examination of the angle of the anterior chamber, using Goldmann threemirror lens) for ocular hypertensive and glaucomatous eyes.

- Dilated fundus examination (using slit lamp biomicroscopy and indirect ophthalmoscopy).

Multimodal imaging procedures including:

(1) Color fundus photography.

(2) Fluorescein angiography.

(3) Optical coherence tomography angiography.

All were performed on the same day or during the same week without in-between intraocular injections or laser treatment.

\section{Optical coherence tomography angiography:}

1- Angio-OCT was done for all patients who were instructed to focus on a fixation target.

2- OCTA images were obtained using the RTVue XR Avanti (AngioVue; optovue Inc, Fremont, California, USA) machine in Bostan Diagnostic Eye Center. 


\section{Ethical approval:}

The study was approved by the medical ethics committee of Al-Azhar University Hospitals and a written informed consent is obtained from all patients.

\section{Statistical analysis:}

Recorded data were analyzed using the statistical package for social sciences, version 20.0 (SPSS Inc., Chicago, Illinois, USA). Quantitative data were expressed as mean \pm standard deviation (SD). Qualitative data were expressed as frequency and percentage.

\section{The following tests were done:}

- Independent-samples t-test of significance was used when comparing between two means.
- Chi-square $\left(\mathrm{x}^{2}\right)$ test of significance was used in order to compare proportions between two qualitative parameters.

- The confidence interval was set to $95 \%$ and the margin of error accepted was set to $5 \%$. The p-value was considered significant as the following:

- Probability (P-value)

- P-value <0.05 was considered significant.

- P-value <0.001 was considered as highly significant.

- P-value >0.05 was considered insignificant.

\section{RESULTS}

The age of our study groups ranged from 15 to 70 years, with an average of $52.47 \pm$ 11.26 years. Ten eyes belonged to 9 males and 9 eyes belonged to 8 females (Table 1)

Table (1): Age \& sex distribution.

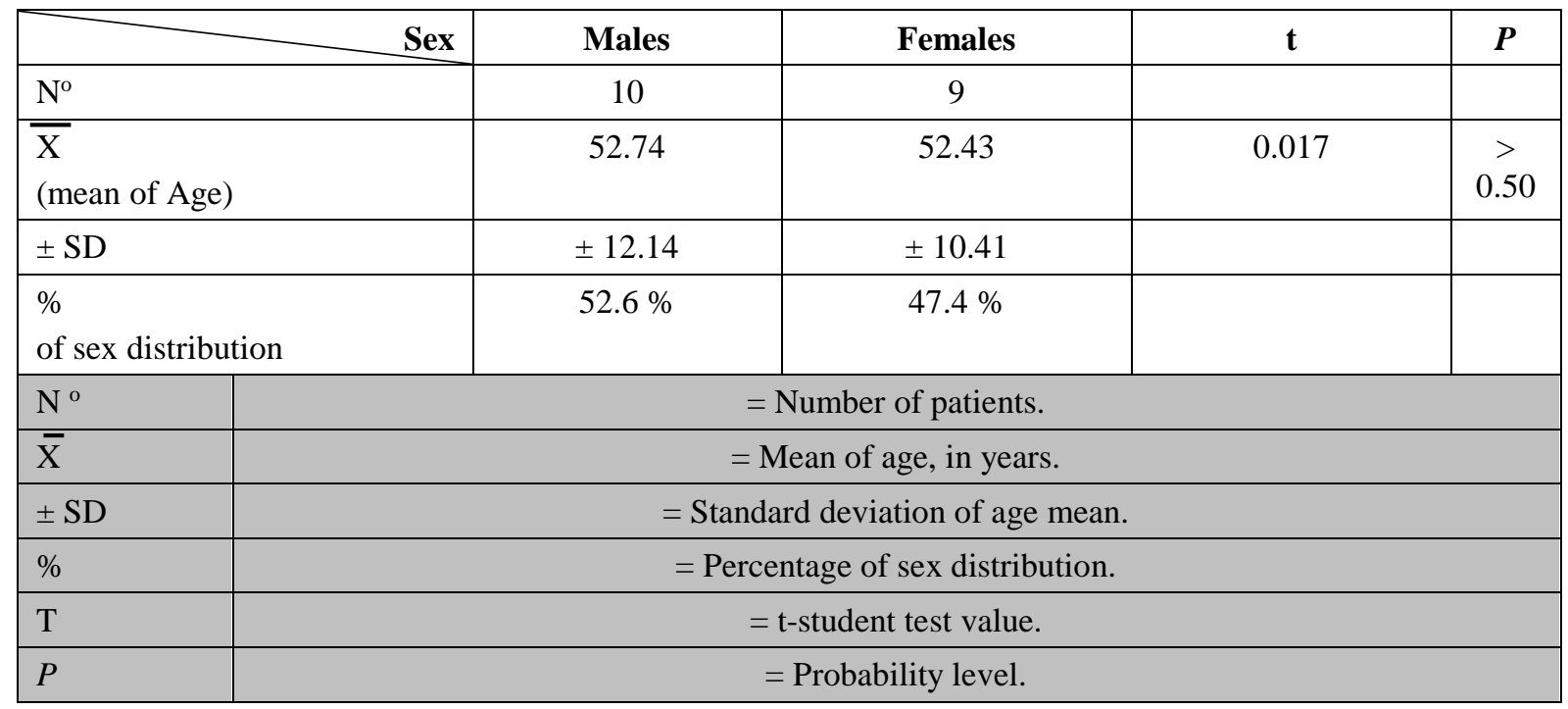

The study also included two forms of CRVO, the classical form which was diagnosed in 15 eyes representing $78.94 \%$ and the Hemi retinal vein occlusion which considered a variant of CRVO and diagnosed in 4 eyes representing $21.05 \%$ (Table 2)

Table (2): Forms of CRVO.

\begin{tabular}{|r|r|}
\hline Form & CRVO \\
\hline
\end{tabular}




\begin{tabular}{|c|c|c|c|}
\hline & Classic & \multicolumn{2}{|c|}{ HRVO } \\
\hline $\mathrm{N}^{0}$ of eyes & 15 & \multicolumn{2}{|c|}{4} \\
\hline$\%$ & $78.94 \%$ & \multicolumn{2}{|c|}{$21.05 \%$} \\
\hline Total $\mathrm{N}^{\mathrm{o}}$ & \multicolumn{3}{|c|}{19} \\
\hline$\%$ & \multicolumn{3}{|c|}{$100 \%$} \\
\hline & & $\mathrm{N}^{\circ}$ & $=$ Number of eyes. \\
\hline CRVO & $=$ Central retinal vein occlusion. & $\%$ & $=$ Percentage \\
\hline HRVO & $=$ Hemiretinal occlusion. & & \\
\hline
\end{tabular}

Relative afferent pupillary defect was diagnosed in 3 eyes of total number and POAG was diagnosed in 3 eyes of the total number of eyes representing $15.79 \%$ of eyes with CRVO while systemic hypertension was found in 7 patients $(40.63 \%)$ (Table 3)

Table (3): Comparison between FA and OCTA regarding the incidence of detected retinal pathological findings of RVO.

\begin{tabular}{|c|c|c|c|c|c|}
\hline \multicolumn{2}{|c|}{ Investigation modality } & FA & OCTA & $\chi^{2}$ & $p$ \\
\hline \multicolumn{2}{|c|}{ Findings $\quad$ CRVO } & CRVO & CRVO & & \\
\hline \multirow[t]{2}{*}{ PFCA visibility } & $\mathrm{N}^{\mathrm{o}}$ & 8 & 18 & \multirow{2}{*}{23.46} & \multirow{2}{*}{$<0.01$} \\
\hline & $\%$ & $42.10 \%$ & $94.74 \%$ & & \\
\hline \multirow[t]{2}{*}{ PFCA disruption } & $\mathrm{N}^{\mathrm{o}}$ & 13 & 19 & \multirow{2}{*}{12.25} & \multirow{2}{*}{$<0.01$} \\
\hline & $\%$ & $68.42 \%$ & $100 \%$ & & \\
\hline \multirow{2}{*}{$\begin{array}{l}\text { Central } \\
\text { Nonperfusion } \\
\text { areas }\end{array}$} & $\mathrm{N}^{\circ}$ & 13 & 19 & \multirow[b]{2}{*}{12.25} & \multirow[b]{2}{*}{$<0.01$} \\
\hline & $\%$ & $68.42 \%$ & $100 \%$ & & \\
\hline \multirow{2}{*}{$\begin{array}{l}\text { Peripheral } \\
\text { Nonperfusion } \\
\text { areas }\end{array}$} & $\mathrm{N}^{\mathrm{o}}$ & 11 & & & \\
\hline & $\%$ & $57.89 \%$ & & & \\
\hline \multirow{2}{*}{$\begin{array}{l}\text { Superficial } \\
\text { capillary } \\
\text { telangiectasia }\end{array}$} & $\mathrm{N}^{\mathrm{o}}$ & 4 & 7 & \multirow[b]{2}{*}{6.72} & \multirow[b]{2}{*}{$<0.01$} \\
\hline & $\%$ & $21.05 \%$ & $36.84 \%$ & & \\
\hline \multirow{2}{*}{$\begin{array}{l}\text { Deep capillary } \\
\text { telangiectasia }\end{array}$} & $\mathrm{N}^{\mathrm{o}}$ & & 19 & & \\
\hline & $\%$ & & $100 \%$ & & \\
\hline \multirow{2}{*}{$\begin{array}{l}\text { Microaneur- } \\
\text { ysms (MA) }\end{array}$} & $\mathrm{N}^{\mathrm{o}}$ & 16 & 19 & \multirow{2}{*}{3.78} & \multirow{2}{*}{$>0.05$} \\
\hline & $\%$ & $84.21 \%$ & $100 \%$ & & \\
\hline \multirow[t]{2}{*}{ Macular edema } & $\mathrm{N}^{\mathrm{o}}$ & 14 & 15 & & \\
\hline & $\%$ & $73.68 \%$ & $78.95 \%$ & & \\
\hline \multirow{2}{*}{$\begin{array}{l}\text { Intraretinal } \\
\text { hemorrhages }\end{array}$} & $\mathrm{N}^{\mathrm{o}}$ & 15 & 15 & & \\
\hline & $\%$ & $78.95 \%$ & $78.95 \%$ & & \\
\hline \multirow[t]{2}{*}{ *Disc edema } & $\mathrm{N}^{\mathrm{o}}$ & 7 & & & \\
\hline & $\%$ & $36.84 \%$ & & & \\
\hline \multicolumn{2}{|l|}{ FA } & \multicolumn{4}{|c|}{$=$ Fluorescein angiography. } \\
\hline \multicolumn{2}{|l|}{ OCTA } & \multicolumn{4}{|c|}{ = Optical coherence tomography Angiography. } \\
\hline \multicolumn{2}{|c|}{\begin{tabular}{|l|l|l} 
CRVO & \\
\end{tabular}} & \multicolumn{4}{|c|}{$=$ Central Retinal vein occlusion. } \\
\hline
\end{tabular}

PFCA Visibility on FA was detected in 8 eyes representing $42.10 \%$ of the total number of eyes compared to 18 eyes representing 94.73 $\%$ were detected by OCTA with a highly significant difference $(\mathrm{P}<0.01)$ between the two modalities. Central nonperfusion areas detected by FA in 13 eyes $(68.42 \%$ ) compared to all eyes $(100 \%)$ showed central nonperfusion areas by OCTA imaging, with a high statistically significant difference $(\mathrm{P}<0.01)$ between the two modalities.

Peripheral nonperfusion areas were only detected by FA. OCTA but could not image peripheral areas of the retina. FA could 
detect peripheral nonperfused areas in 11 eyes representing 57.89 of eyes with CRVO.

Superficial Capillary telangiectasia was observed by FA in 4 eyes representing $21.05 \%$ and in 7 eyes by OCTA representing $36.84 \%$ with high statistically significant difference $(\mathrm{P}$ $<0.01$ ) between the two modalities. Deep capillary telangiectasia revealed only by OCTA in all eyes $(100 \%)$. Microaneurysms revealed in 16 eyes by FA $(84.21 \%)$ and in all eyes (100 $\%)$ by OCTA. However, the statistical difference was found non-significant $(\mathrm{P}>0.05)$. Macular edema revealed in 14 eyes $(73.68 \%)$ by both FA and OCTA (with the help of the co- registered en face OCT) modalities.

However, B-Scan OCT could detect macular edema in 17 eyes representing $89.47 \%$ of the total number of eyes. Intraretinal hemorrhages were detected equally by both FA \& OCTA in 15 eyes with CRVO representing $78.9 \%$. Disc edema was only detected by FA which could detect disc leakage in 7 eyes $(36.84$ $\%$ ) of the total number of eyes with CRVO. All of above result show difference between FFA and OCTA fining in CRVO (table 4). The following result show comparison between incidence of pathological retinal changes of SCP and those of DCP in RVO as revealed by OCTA (Table 4, 5).

Table (4): Comparison between superficial capillary plexus and deep capillary plexus regarding the incidence of detected retinal pathological findings of CRVO.

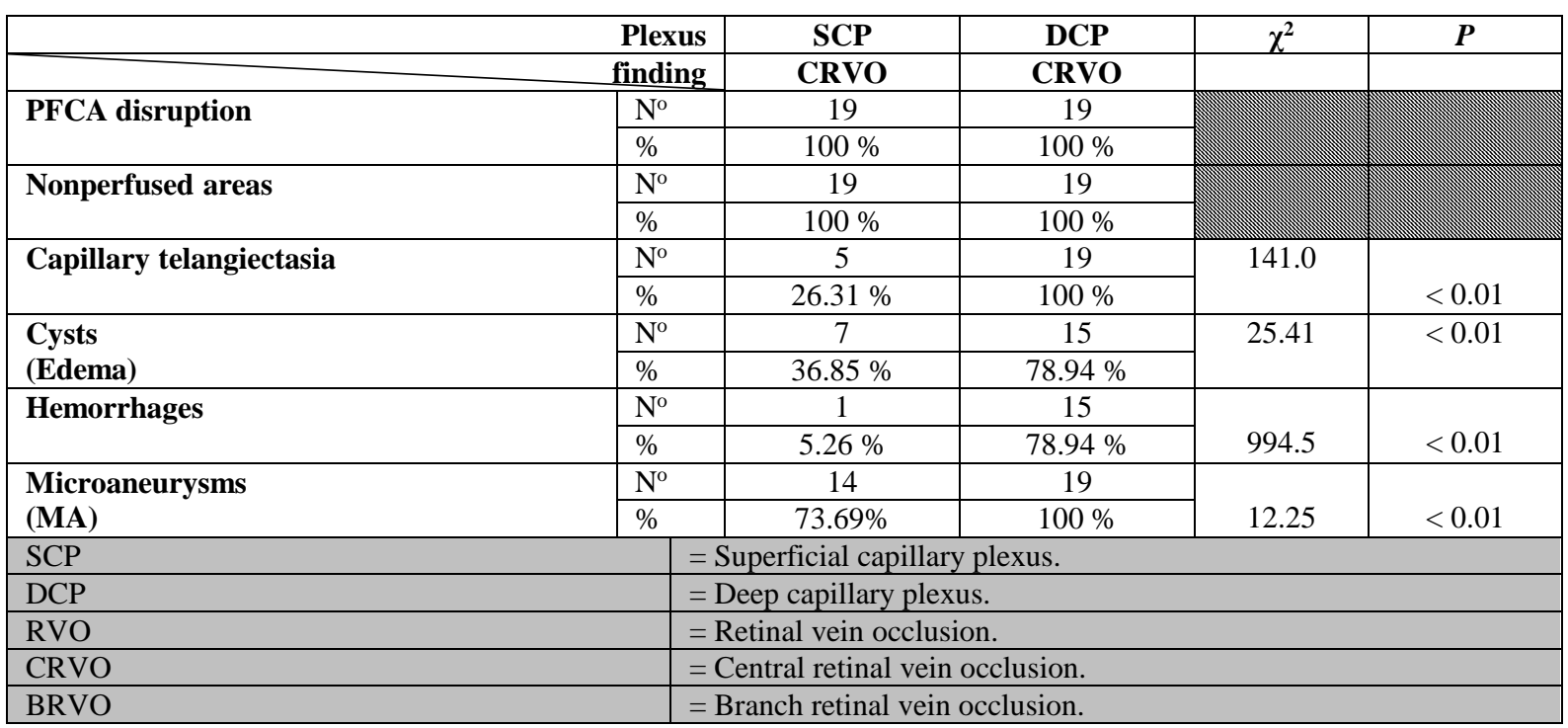

Table (5): The mean vessel area density percentage of RVO, CRVO and BRVO as compared to normal control and comparison of mean vessel area density percentage between CRVO and BRVO.

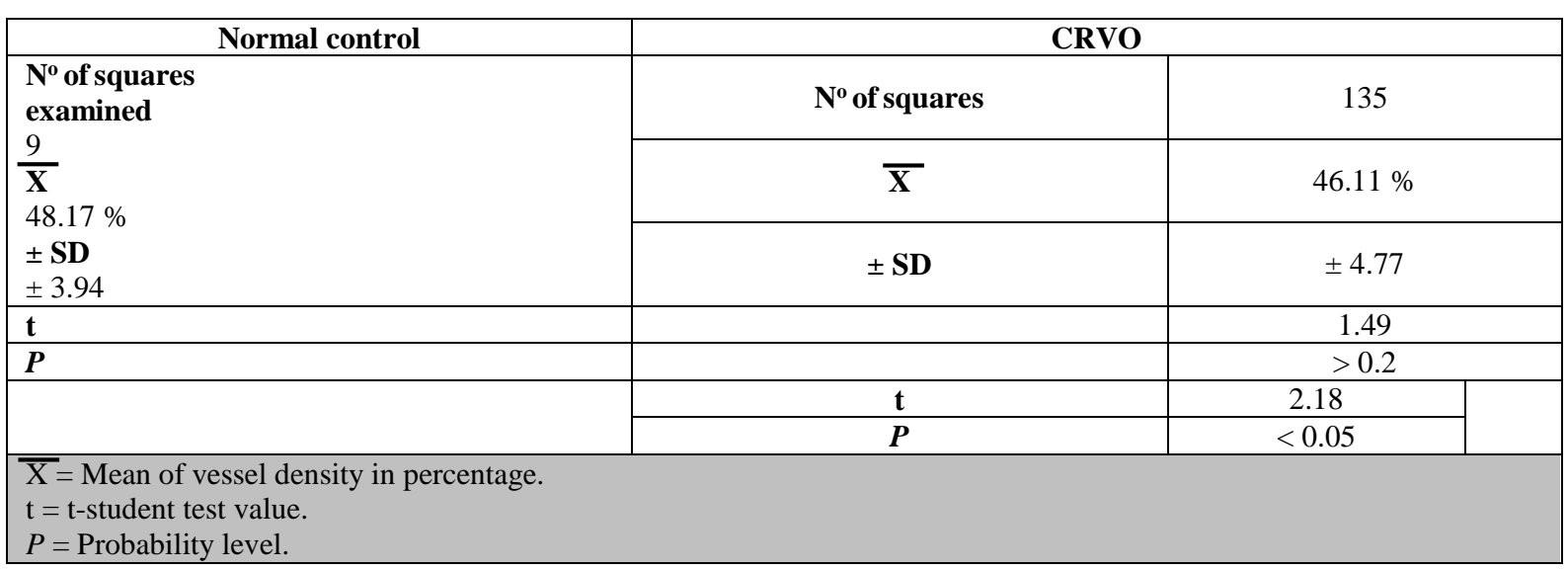

Perifoveal capillary disruption was revealed in both SCP and DCP of all eyes of the study. Nonperfusion areas again revealed in both SCP and DCP of all eyes. 
Capillary telangiectasia was revealed in SCP of only 5 eyes $(26.31 \%)$ compared to its detection in the DCP of all eyes $(100 \%)$ with high significant difference $(\mathrm{P}<0.01)$ of their detection between the two plexa.

Cysts (edema) in SCP, were detected (on OCTA with the help of the coregistered en face OCT) in 7 eyes (36.84 \%) and in DCP were detected (on OCTA with the help of the coregistered en face OCT) in 15 eyes $(78.94 \%)$ with statistically significant difference $(\mathrm{P}<$ $0.01)$ between the two plexa. Hemorrhages were detected in SCP in only one eye $(5.26 \%)$ compared to 15 eyes revealing hemorrhages in DCP (78.95 \%) with high statistically significant difference $(\mathrm{P}<0.01)$ of their occurrence between the two plexa. Microaneurysms Detected in SCP of 14 eyes $(73.69 \%)$ and in DCP of all eyes (100\%) with high statistically significant difference $(\mathrm{P}<$ 0.01 ) of incidence of their detection between the two plexa.

\section{Discussion}

Martinez et al. ${ }^{(1)}$ stated that RVO is the most common retinal vascular disease after diabetic retinopathy. It affects not only elderly individuals but also young patients ${ }^{(8)}$. Hayreh et al. (9) found that CRVO occurred predominately over the age of 65 years and only $10 \%$ occurred before 40 years of age. Lahey $\boldsymbol{e t}$ al. ${ }^{(10)}$ concluded that RVO was rare under 30 years of age. In our study, age range was 15-70 years, the mean age was $52.47 \pm 11.26$ for the total number of patients $(\mathrm{n}=17)$, and according to sex: the mean age of males was $52.74 \pm 12.14$ years and of females $52.43 \pm 10.41$ years with no significant difference $(\mathrm{t}=0.17, \mathrm{P}>0.50)$ between the two sexes.

Marcucci et al. ${ }^{(11)}$ stated that POAG is the most frequent ocular disorder predisposing to RVO mainly CRVO and to a lesser extent BRVO. Our study revealed POAG in $15.79 \%$ of CRVO. Lee et al. ${ }^{(12)}$ concluded that diabetes is strongly associated with CRVO. Our study, however, revealed association of systemic hypertension with CRVO in $35 \%$ of the total number of patients and in considering association of RVO with diabetes, diabetic patients were excluded from our study. In our study, perifoveal capillary arcade (PFCA) and central nonperfusion areas (CNPA) were more observable on OCTA images than those of FA, as concluded by the high statistically significant difference $(\mathrm{P}<0.01)$ of the total number of eyes in both parameters between the two modalities.

These observations were also pointed out by Suzuki et al. ${ }^{(4)}$ and Coscas et al. ${ }^{(7)}$ and could be explained by the fact that, PFCA and CNPA were masked in FA images by intraretinal hemorrhages which induced hypofluorescence and/or leaking vessels which induced hyperfluorescence. Disruption of PFCA of SCP was observed much more frequently $(\mathrm{P}<0.01)$ in images of OCTA than in those of FA. PFCAD was revealed in both SCP and DCP of all eyes of this study on OCTA. Coscas et al. (7), however, reported higher incidence of PFCAD in DCP than in SCP, but with no statistically significant difference $(\mathrm{P}=0.50)$. On OCTA retinal ischemia is expressed as areas of nonperfusion whether central or peripheral which correspond to area of capillary dropout on FA images.

Central nonperfusion areas (CNPA) were seen on OCTA as hypointense, dark grayish areas with reduced capillary density and capillary network disruption in both superficial and deep capillary plexuses ${ }^{(4)}$. In our study, OCTA images visualized CNPA clearer than the corresponding areas of capillary dropout on FA images, thus OCTA was superior in delineating CNPA and differentiating them from the FAZ and also from the surrounding perfused retina Kashani et al. ${ }^{(3)}$ and Suzuki $\boldsymbol{e t}$ al. ${ }^{(4)}$ reported the same observation. CNPA were revealed in both SCP and DCP of all eyes of our study.

Our study agreed with the study of Cardoso et al. ${ }^{(13)}$ who reported no notable difference in capillary nonperfusion between the two plexa. On the other hand, Coscas et al. (7) reported more frequent NPA in DCP than in SCP statistically $(\mathrm{P}<0.001)$. Peripheral nonperfusion areas (PNPA) are only detected by FA. OCTA couldn't detect peripheral nonperfusion areas as it cannot image peripheral areas of the retina. The important retinal vascular changes in RVO include: Telangiectasia (Superficial and deep); microaneurysms and collateral vessels. In our study, superficial capillary telangiectasia (SCT), which are capillary dilatations appeared, on OCTA, as multiple intraretinal loops with hyper signal in the SCP, they were also observed in FA images, however, the number of eyes in which SCT was detected is statistically higher than those detected on FA images. Besides, OCTA could visualize SCT clearer than did FA, not only that, but also the normal 
tiny details of the capillary network in the SCP.

Suzuki et al. ${ }^{(4)}$ registered the same observations. The superiority of OCTA modality in detecting telangiectasia is due to its higher resolution than FA modality, and also due to less affection with media opacities ${ }^{(4,14)}$. In our study, deep capillary telangiectasia (DCT), like SCT, could be seen as capillary dilatation with hyper signal on OCTA images. All eyes showed this pathological entity by only OCTA modality. Same observation was reported by Suzuki et al. ${ }^{(4)}$.

On FA images, deep capillary telangiectasia was detected; as FA is a twodimensional imaging modality ${ }^{(4)}$. In our study, deep capillary telangiectasia (DCT), like SCT, was seen as capillary dilatation with hyper signal on OCTA images. All eyes showed this pathological entity by only OCTA modality. Same observation was reported by Suzuki et al. (4). On FA images, deep capillary telangiectasia couldn't be detected; as FA is a two-dimensional imaging modality ${ }^{(4)}$. The presence of microaneurysms seems to be one of the changes of retinal microvasculature in cases of CRVO Several mechanisms have been postulated to explain their pathogenesis: Schulman et al. ${ }^{(15)}$ attributed their development to loss of pericytes of capillary walls leading to their weakness similar to what occurs in diabetic retinopathy leading to their formation. Many other authors as Bowers et al. ${ }^{(16)}$, De Paola et al. ${ }^{(17)}$ and Kumar et al. (18) attributed micraneurysm formation to compression of the veins leading to increased retinal venous blood flow velocity with shear stress of blood on vascular endothelium resulting in reactive endothelial cell proliferation and damage with final microaneurysmal formation.

In our study, OCTA could detect microaneurysms in all eyes compared to only 16 eyes in which microaneurysms were detected by FA modality, however, no statistical significant difference $(\mathrm{P}>0.05)$ was found between both modalities in this respect. Suzuki et al. ${ }^{(4)}$ reported almost equal capability of both modalities in detecting microaneurysms, with only one eye with microaneurysms which was detected on FA and not on OCTA. The more severe affection of DCP with microaneurysms in RVO has been explained by Moore et al. ${ }^{(19)}$ and Browning (20), who stated that microaneurysms seem to be a microvascular response to vascular endothelial growth factor (VEGF) which has been generated from hypoxic retinal tissue and since SCP as stated by Martinet et al. ${ }^{(21)}$ has higher perfusion pressure and oxygen supply in RVO, thus it is better preserved than DCP. On the other hand, our study demonstrated that microaneurysms were detected mainly around NPA in SCP, and in the walls of telangiectatic vessels around cystoid spaces in DCP, and thus microaneurysms may be implicated in the pathogenesis of macular edema. Same observation and explanation were concluded by Suzuki et al. ${ }^{(4)}$, where collateral vessels were detected by both FA and OCTA modalities in the same two eyes. This was in agreement with Suzuki et al. ${ }^{(4)}$, who detected collateral vessels equally by OCTA and FA modalities.

Rispoli et al. ${ }^{(22)}$ found that OCTA was better than FA in visualizing collateral vessels. Our study as well as the study of Suzuki $\boldsymbol{e t}$ al. (4) revealed the superiority of OCTA over FA as it facilitates differential analysis of collateral vessels. Collateral vessels, in our study were detected in the SCP of the two eyes, in contrary to the report of Suzuki et al. ${ }^{(4)}$ who detected them in both plexa. However, both studies revealed collaterals at the margins or around NPA in images of both OCTA and FA modalities, and this location might support the pathogenesis of collateral vessels' existence described by Dithmar and Holz ${ }^{(23)}$. Intraretinal edema was diagnosed on FA images as areas of hyper-fluoresscence in late-phase. It could have a petaloid pattern (or clover-leaf like pattern) in cases of cystoid macular edema ${ }^{(23)}$.

On OCT B-scan, edema was seen as a hypo-reflective pockets with increased retinal thickness ${ }^{(24)}$. Our study demonstrated edema in the eyes of the study series by both modalities; FA and OCT B-scan. Besides both modalities, our study could detect intraretinal edema on OCTA as well-defined black roundish cysts with no decorrelation flow signal corresponding to intraretinal cystoid spaces (meshwork changes or lesions), on the co-registered en-face OCT images which helped us to detect the exact lateral extension (circumference) of the edema Our observation was in agreement with that of Coscas et al. ${ }^{(7)}$, who could detect intraretinal cystoid spaces on OCTA with the help of the coregistered en face OCT images. However, Kashani et al. ${ }^{(3)}$ failed to detect intraretinal edema on OCTA images in any of the examined eyes and concluded that OCTA could detect only chronic appearing intraretinal edema that was typically associated with hard exudates 
which appear as hyper-reflective foci on SD OCT Bscans. Intraretinal hemorrhage, as a finding of CRVO, is diagnosed on FA as areas of hypofluoresence Rispoli et al. ${ }^{(22)}$ and on Bscan OCT as intraretinal hyper-reflectivity ${ }^{(25)}$.

Our study demonstrated intraretinal hemorrhages on both FA and B-scan OCT. Besides both modalities, our study demonstrated intraretinal hemorrhages on OCTA as ill-defined black areas with no decorrelation flow signal, corresponding to the low reflectivity patches seen on the coregistered en-face OCT images, where these patches were produced on the en face images due to attenuation of the OCT signal. Same findings were detected by Kashani et al. ${ }^{(3)}$.

Although, intraretinal hemorrhages were detected equally by both FA and OCTA, as demonstrated by our study and that of Kashani et al. ${ }^{(3)}$, OCTA (together with the en face OCT), however, could clarify the level of hemorrhage existence within the retina. On this respect, our study revealed intraretinal hemorrhages in the DCP of 15 eyes and in both plexuses in one of them with statistically significant difference $(\mathrm{P}<0.01)$ between the incidence of hemorrhages in DCP compared to that in SCP (Table 5). In our study series, disc edema without NVD could only be detected by FA which could detect disc leakage in 7 eyes with disc edema. OCTA could not detect disc leakage in these 7 eyes with disc edema, but there were some findings on OCTA rather than disc leakage, that helped us to detect disc edema in these eyes. Our study introduces additional advantage of the OCTA over the conventional FA imaging modality in RVO; which is the ability of OCTA to perform quantitative flow analysis of the retinal vasculature in CRVO.

The previous studies which have used OCTA to quantify retinal capillary density and the foveal avascular zone area, were done on either healthy subjects; in order to find out age matched normative data of the microvascular density and the FAZ area of both the SCP and the DCP, or patients with diabetic retinopathy; in order to compare their microvascular density with age-matched controls. One of these studies that was done on healthy subjects; is the study that was conducted by Shahlaee $\boldsymbol{e t}$ al. ${ }^{(26)}$ who reported mean parafoveal vascular densities of $46 \%$ and $52 \%$ in the superficial vascular network and the deep vascular network, respectively and also reported negative correlations with age. In our study, we found that the degree of ischemia involving the SCP, can be appreciated quantitatively with:

1. Non-Flow measurement which is operator dependent and may lead to false estimation of the ischemia.

2. Vessel density measurements, where the vessel density is calculated as the percentage area occupied by vessels in the selected region (27), and can be appreciated qualitatively with the color coded vessel density maps; where the areas of severe ischemia are color coded as dark blue. The statistical analysis of the grid-based vessel area density percentages that was done in our study, proved that the RVO is injurious to the retinal vasculature of the SCP (the mean value of the normal control $=48.17 \pm 3.94$ (the mean value of CRVO group $=46.11 \pm$ $4.77 \%)$

\section{Conclusion}

Our study has demonstrated that optical coherence tomography angiography can qualitatively demonstrate most of the pathological findings of central retinal vein occlusive disorder and all of its macular complications including; ischemia, capillary dilation, microaneurysms, collateral vessels, edema and intraretinal hemorrhages, with superiority to fluorescein angiography especially in the presence of retinal hemorrhages and retinal edema.

It is well known that fluorescein angiography has the disadvantage of being invasive; depending on a dye with possible occurrence of adverse reactions. Also, this dye which should provide important in formation, can impair the visualization of the capillary abnormalities and areas of capillary dropouts, which we found in our study. On the other hand, we found that fluorescein angiography is a very useful and essential tool for evaluation and diagnosis of peripheral retinal involvement, mainly peripheral ischemia.

The second main advantage is that it allows for stratigraphic analysis of retinal and choroidal vasculatures, providing images for superficial capillary plexus, deep capillary plexus, outer retina and choriocapillaris, i.e. it provides new insights in imaging the deep capillary plexus which couldn't be possible previously. The importance of this stratigraphic analysis is the potential differential involvement of the two retinal capillary plexuses in most retinal vascular diseases and as our study 
demonstrated, more severe involvement of the deep capillary plexus in retinal vein occlusion.

The third advantage is that it performs quantitative flow analysis of the retinal vasculature through the vessel density measurements. The values of the superficial and deep capillary plexuses' vessel density can provide an easily interpretable noninvasive objective tool for quantitative evaluation of the retinal perfusion status which will facilitate the management and monitoring the progression of different retinal vascular disorders including, retinal vein occlusion.

The quantitative flow analysis that we have performed in our study, involved only the superficial capillary plexus and did not take into consideration the age of the patients selected for flow analysis where previous studies on healthy human eyes, reported negative correlation of the superficial and deep capillary plexuses' vessel density with age. So future directions that we recommend for this study, should include performing the quantitative flow analysis on both the superficial and the deep capillary plexuses in retinal vein occlusion, taking into consideration; the age of the study population, the duration of retinal vein occlusion disease, whether it is previously treated or not, type of treatment; whether retinal laser photocoagulation, intravitreal injection or both.

\section{REFERENCES}

1. Martinez F, Furio E, Fabia MJ et al. (2014): Risk factors associated with retinal Vein Occlusion. Int J Clin Pract., 68(7): 871-881.

2. Cugati S, Wang JJ, Rochtchina E et al. (2006): Ten-Year incidence of retinal emboli in an older population. Stroke, 37: 908-10.

3. Kashani AH, Lee SY, Moshfeghi A et al. (2015): Optical coherence tomography angiography of retinal venous occlusion. Retina, 35: 23232331.

4. Suzuki N, Hirano Y, Yoshida M et al. (2015): Microvascular abnormalities on optical coherence tomography angiography in macular edema associated with branch retinal vein occlusion. Am J Ophthalmol., 161: 126-132.
5. De Carlo TE, Salz DA, Waheed NK et al. (2015): Visualization of the retinal vasculature using Wide- Field Montage Optical Coherence Tomography Angiography. Ophthalmic Surg Lasers Imaging Retina, 46: 611-616.

6. Spaide RF, Klancnik JM and Cooney MJ (2015): Retinal vascular layers imaged by Fluorescein Angiography and Optical Coherence Tomography Angiography. JAMA Ophthalmology, 133(1): 45-50.

7. Coscas F, Glacet-Bernard A, Miere A et al. (2016): Optical Coherence Tomography Angiography in retinal vein occlusion: Evaluation of superficial and deep capillary plexa. Am J Ophthalmol., 161: 160-171.

8. Yioti GG, Panagiotou OA, Vartholomatos GA et al. (2013): Genetic polymorphisms associated with retinal vein occlusion: A Greek case-control study and meta-analysis. Ophthalmic Genetics, 34(3): 130-139.

9. Hayreh SS, Zimmerman MB, Podhajsk P (1994): Incidence of various types of retinal vein occlusion and their recurrence and demographic characteristics. Am J Ophthalmol., 117: 429-441.

10. Lahey JM, Kearney JJ, Tunc M (2003): Hypercoagulable states and central retinal vein occlusion. Curr Opin Pulm Med., 9: 385- 392.

11. Marcucci R, Bertini L, Giusti B et al. (2001): Thrombophilic risk factors in patients with central retinal vein occlusion. Thromb Haemost., 86: 772776.

12. Lee JY, Yoon YH, Kim HK et al. (2013): Baseline characteristics and risk factors of retinal vein occlusion: A Study by the Korean RVO Study group. J Korean Med Sci., 28: 136-144.

13. Cardoso JN, Keane PA, Sim DA et al. (2016): Systematic evaluation of Optical Coherence Tomography Angiography in retinal vein occlusion. Am J Ophthalmol., 163: 93-107. 
14. Kawasaki R, Nagano E, Uno M et al. (2013): Retinal vascular features associated with risk of branch retinal vein occlusion. Current Eye Research, 38(9): 989-993.

15. Schulman J, Jampol LM, Goldberg MF (1981): Large capillary aneurysms secondary to retinal venous obstruction. Br J Ophthalmol., 65(1): 36-41.

16. Bowers DK, Finkelstein D, Wolff SM and Green WR (1987): Branch retinal vein occlusion: a clinicopathologic case report. Retina, 7(4): 252-259.

17. De Paola N, Gimbrone MA, Davies PF et al. (1992): Vascular endothelium responds to fluid shear stress gradients. Arterioscler Thromb., 12 (11): 12541257.

18. Kumar B, Yu DY, Morgan WH et al. (1998): The distribution of angioarchitectural changes within the vicinity of the arteriovenous crossing in branch retinal vein occlusion. Ophthalmology, 105(3): 424-427.

19. Moore J, Bagley S, Ireland G et al. (1999): Three dimensional analysis of microaneurysms in the human diabetic retina. J Anat., 194(1): 89-100.

20. Browning DJ (2012): Pathophysiology of Retinal Vein Occlusions" In "Retinal Vein Occlusions: Evidence-Based Management", edited by Browning DJ, New York: Springer. Chap 2, Pp 33-72.

21. Martinet V, Guigui B, GlacetBernard A et al. (2012): Macular optical coherence tomography, fluorescein angiography and visual acuity. Int Ophthalmol., 32(4): 369377.

22. Rispoli M, Lumbroso $B$ and Savastano MC (2015): OCT angiography of vascular occlusions in Clinical OCT Angiography Atlas, edited by Lumbroso B, Huang D, Chen CJ et al., Chap 14, Pp. 112-119.

23. Dithmar S and Holz FG (2007): Fluorescence Angiography in Ophthalmology. Chap 1, The physical and chemical fundamentals of fluorescence angiography, Springer. pp $1-3$,

24. Duker JS, Waheed NK, Goldman DR (2014): Handbook of Retinal OCT". Chap 14.1, "Branch Retinal Vein Occlusion", EL Sevier, Saunders. Pp. 92-93,

25. Yuan A and Kaiser PK (2013): Branch Vein Occlusion In Retina, edited by Ryan SJ, 5th edition, Chap 53, pp 1029-1038, Elsevier, Saunders.

26. Shahlaee A, Samara WA, Hsu J et al. (2016): In Vivo assessment of macular vascular density in healthy human eyes using optical coherence tomography angiography. Am J Ophthalmol., 165: $39-46$.

27. Huang D and Jia Y (2015): SplitSpectrum Amplitude- Decorrelation Angiography In Clinical Guide to Angio-OCT , edited by Lumbroso B, Huang D, Jia Y et al., Jaypee Brothers Medical Publishers (P) Ltd, Chap 3, pp 8-9, 